\title{
Images From Headache
}

\section{Resolution of Trigeminal Mediated Nasal Edema Following Greater Occipital Nerve Blockade}

\author{
Wade Cooper, DO
}

A 45-year-old man with a history of hypertension and no tobacco exposure presented with a 13-year history of daily strictly right-sided continuous headache that began 2 months after concussion. His headache was associated with mild photophobia, phonophobia, nausea, and rare episodes of emesis as well as constant right-sided nasal stuffiness for "years." Additionally, the patient would experience severe exacerbation of headache with associated right-sided trigeminal autonomic features including ptosis, lacrimation, rhinorrhea, and agitation lasting 20-30 minutes. Although a diagnosis of chronic paroxysmal hemicrania was considered, the patient did not respond to indomethacin at $150 \mathrm{mg} /$ day.

Brain magnetic resonance imaging (MRI) performed on March 2, 2007 revealed increased T2 signal on axial imaging throughout the right-sided nasal mucosa (Fig. 1). The patient received a right-sided occipital nerve block on March 8, 2007 and achieved pain relief within minutes. Additionally, the patient stated "I can finally breathe" referring to resolution of his constant right-sided nasal stuffiness. Repeat axial T2 MRI performed on March 10, 2007 revealed resolution of the nasal mucosal edema identified on previous imaging (Fig. 2).

Greater occipital nerve (GON) blockade with local anesthetic and corticosteroid has been widely used in clinical practice for many years. GON blockade has been shown to be effective in many primary headache disorders including migraine, cluster, hemi-

From the University of Michigan-Neurology and Anesthesiology, Ann Arbor, MI, USA.

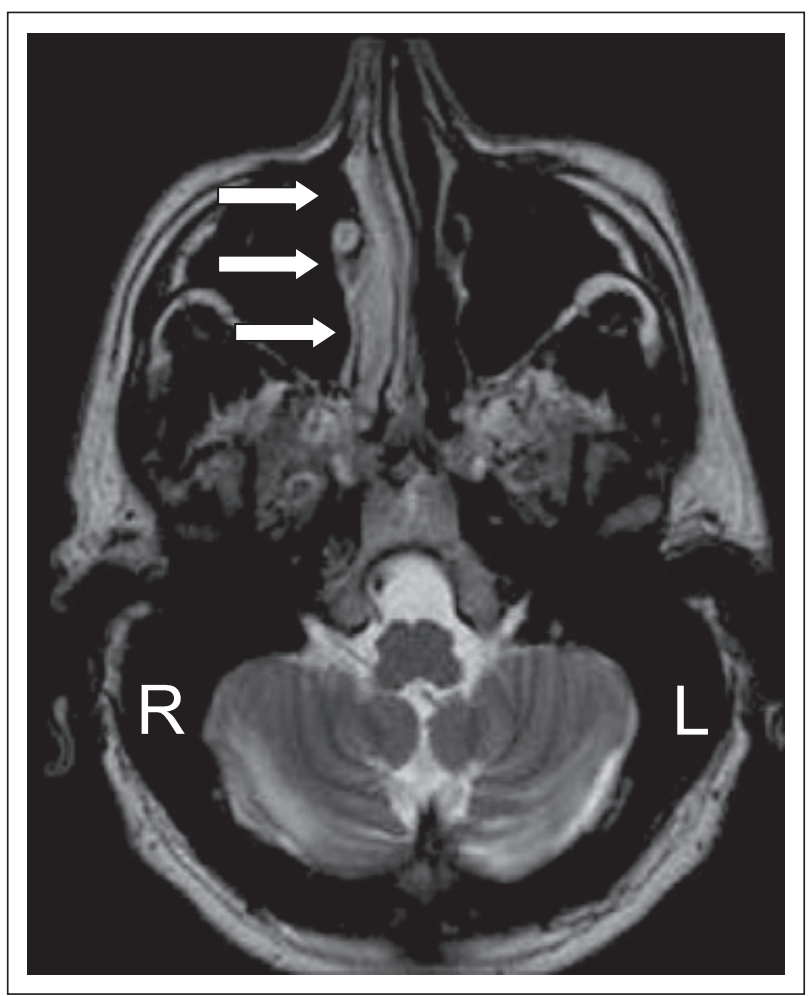

Fig 1.-T2-weighted magnetic resonance imaging with increased T2 signal throughout the right nasal mucosa (arrows).

crania continua, and cervicogenic headache. Tenderness over the GON region to manual palpation has been found to be predictive of clinical response to regional blockade. ${ }^{1}$ It has been hypothesized that the GON blockade may suppress the trigeminal activity via the spinal trigeminal nucleus and consequently affect the trigeminal autonomic pathways. ${ }^{2,3}$ In this case, it is hypothesized that GON blockade not only 




Fig 2.-T2-weighted magnetic resonance imaging with symmetric normal appearing nasal mucosa following occipital nerve block (arrows). relieved pain, but also resolved the patient's trigeminal autonomic feature of nasal stuffiness as evidenced by MRI.

\section{REFERENCES}

1. Afridi SK, Shields KG, Bhola R, Goadsby PJ. Greater occipital nerve injection in primary headache syndromes - Prolonged effects from a single injection. Pain. 2006;122:126-129.

2. Peres MFP, Stiles MA, Siow HC, Rozen TD, Young WB, Silberstein SD. Greater occipital nerve blockade for cluster headache. Cephalalgia. 2002;22:520-522.

3. Goadsby PJ, Bahra A, May A. Mechanisms of cluster headache. Cephalalgia. 1999;19(Suppl. 23):19-21. 